\title{
The temporal dynamics of metacognition: Dissociating task-related activity from later metacognitive processes
}

\author{
Kobe Desender ${ }^{\mathrm{a}, *}$, Filip Van Opstal ${ }^{\mathrm{b}, \mathrm{c}}$, Gethin Hughes ${ }^{\mathrm{d}}$, Eva Van den Bussche ${ }^{\mathrm{a}}$ \\ a Department of Psychology, Vrije Universiteit Brussel, Brussels, Belgium \\ ${ }^{\mathrm{b}}$ Center for Research on Cognition \&' Neurosciences (CRCN), Université Libre de Bruxelles (ULB), Belgium \\ ${ }^{\mathrm{c}}$ ULB Neurosciences Institute (UNI), Belgium \\ ${ }^{\mathrm{d}}$ Department of Psychology, University of Essex, United Kingdom
}

\section{A R T I C L E I N F O}

\section{Article history:}

Received 21 August 2015

Received in revised form

27 November 2015

Accepted 4 January 2016

Available online 8 January 2016

\section{Keywords::}

Response conflict

Metacognition

N2/P3

Precuneus

ACC

EEG

\begin{abstract}
A B S T R A C T
In recent years, neuroscience research spent much effort in revealing brain activity related to metacognition. Despite this endeavor, it remains unclear exactly when metacognitive experiences develop during task performance. To investigate this, the current study used EEG to temporally and spatially dissociate task-related activity from metacognitive activity. In a masked priming paradigm, metacognitive experiences of difficulty were induced by manipulating congruency between prime and target. As expected, participants more frequently rated incongruent trials as difficult and congruent trials as easy, while being completely unable to perceive the masked primes. Results showed that both the N2 and the P3 ERP components were modulated by congruency, but that only the P3 modulation interacted with metacognitive experiences. Single-trial analysis additionally showed that the magnitude of the P3 modulation by congruency accurately predicted the metacognitive response. Source localization indicated that the N2 task-related activity originated in the ACC, whereas the P3-interplay between taskrelated activation and metacognitive experiences originated from the precuneus. We conclude that taskrelated activity can be dissociated from later metacognitive processing.
\end{abstract}

(c) 2016 Elsevier Ltd. All rights reserved.

\section{Introduction}

When acting upon stimuli in the environment, our actions are accompanied by metacognitive experiences. For example, when typing on a foreign keyboard, you will clearly experience that your actions do not proceed very fluently. While the neurocognitive underpinnings of metacognition have recently received a lot of attention (Fleming et al., 2014, 2010; McCurdy et al., 2013), it remains unclear how these metacognitive experiences develop in time. For example, it is highly debated whether metacognitive experiences associated with our actions are created at the same time of the decision to act, or whether they also depend on new information arriving beyond this decision point (Yeung and Summerfield, 2012). More generally, it is unknown at which point in time specific neural processes contribute to the creation of metacognitive experiences.

Metacognition, a general term used to describe the subjective experiences associated with our actions, has been studied in a

\footnotetext{
* Correspondence to: Faculty of Psychology and Educational Sciences, Vrije Universiteit Brussel, Pleinlaan 2, 1050 Brussels, Belgium.

E-mail address: Kobe.Desender@vub.ac.be (K. Desender).
}

variety of research fields. In the meta-memory literature, researchers have extensively investigated subjective experiences associated with memory formation, such as judgments-of-learning during acquisition (Metcalfe and Finn, 2008), and feeling-ofknowing during recall (e.g., Díaz et al., 2007). In the neurocognitive literature, most studies use low-level perceptual decision tasks, and examine the degree of confidence associated with decisions (de Gardelle and Mamassian, 2014; Fleming and Lau, 2014; Fleming et al., 2010) or the awareness of having made an error in the decision process (Boldt and Yeung, 2015; Steinhauser and Yeung, 2010). In the current study, we focus on one particular class of metacognitive experiences, namely the experience of fluency in action-selection. Several recent studies already demonstrated that participants can reliably introspect on the fluency of their actionselection, even when they are unaware of the stimuli manipulating the fluency of selection. For example, Charles et al. (2013) showed that participants could differentiate between correct and incorrect judgments in a simple decision task, even though they did not perceive the stimulus they had to decide on. In a similar vein, studies have used subliminal priming to create a conflict between two responses, and observed that task performance and perceived difficulty were jointly influenced, without participants being 
aware of the visual stimuli driving these changes (Chambon and Haggard, 2012; Desender et al., 2014; Wenke et al., 2010). That is, even though participants are completely unaware of the presence of the subliminal stimuli creating the response conflict, they nevertheless have the metacognitive experience that responding was more difficult on those trials where the subliminal stimulus interfered with response selection. A major benefit of these conflict paradigms is that a large body of research has already documented the neural components associated with the processing of response conflicts. However, while it was recently demonstrated that metacognitive experiences are critically involved in conflict processing (Desender et al., 2014), the role of metacognitive experiences in relation to these components has not been studied.

Conflict tasks are known to reliably modulate two important event-related components (ERPs) in the EEG waveform (Ullsperger et al., 2014). First, a fronto-central N2 component around 200$300 \mathrm{~ms}$ post-stimulus is observed, which is believed to reflect a sensitivity of the anterior cingulate cortex (ACC) to the activation of incompatible responses (Van Veen and Carter, 2002). Later in time, a central-parietal $\mathrm{P} 3$ component around $300-400$ ms post-stimulus emerges, whose functional role is still a matter of debate. Some consider it to be an index of stimulus evaluation (Coles et al., 1985; Purmann et al., 2011), whereas others assume that the P3 reflects the engagement of attentional resources needed for improved control (Clayson and Larson, 2011; West, 2003). Interestingly, the P3 component is also considered as a signature for conscious access (Del Cul et al., 2007; Kouider et al., 2013), making it a likely neural correlate of metacognitive awareness in conflict tasks.

Based on existing evidence, both the N2 and P3 could be involved in metacognition. First, noting that activity in the ACC is related to both cognitive (e.g., response conflict, errors) and affective (e.g., pain) factors, Spunt et al. (2012) showed that the ACC tracks changes in subjective experience, such as frustration and negative affect (for theoretical perspectives, see e.g., Hillman and Bilkey (2013) and Shackman et al. (2011)). Therefore, metacognition could be related to activity in the ACC, reflected by the N2 component. Second, research on error processing revealed that awareness of one's own errors selectively modulates the error positivity (Pe) around $300 \mathrm{~ms}$ post-response (Hughes and Yeung, 2011; Nieuwenhuis et al., 2001), whereas the earlier error related negativity (ERN; originating from the ACC; Yeung et al., 2004) is only modulated by objective accuracy (although this latter claim has been contested, see e.g., Scheffers and Coles, (2000), Shalgi and Deouell, (2012) and Wessel, (2012)). Given that the Pe is considered to be the error-related homolog of the P3 (Ridderinkhof et al., 2009), metacognition should be expressed in the P3 component only.

In short, while both the N2 and the P3 could theoretically be linked to metacognitive experiences, this has not been tested before. The aim of the current study is to investigate this and to dissociate task-related activity from activity related to metacognitive experiences.

\section{Materials and methods}

\subsection{Participants}

Thirty-one participants, 17 female and 14 male, participated in return for a monetary compensation (£15). Mean age of the sample was 24.3 years $(S D=5.2$, range $19-42)$. All participants were right-handed, had normal or corrected-to-normal vision, had no history of epilepsy and were not taking psychoactive drugs. The study was approved by the Ethics Committee of the University of Essex and written informed consent was obtained from each participant prior to the experimental session. Because of intense sweating, caused by extreme hot weather conditions, and resulting noise on the EEG recordings, the data of six participants were unfit for analyses. The data of one additional participant were excluded because of technical problems with the EEG recording.

\subsection{Experimental procedure}

Participants were seated in a dimly lit room for the duration of the experimental session. Participants completed a masked priming experiment in which they additionally were asked to report about the metacognitive experience associated with their response (see Fig. 1). Each experimental trial started with a fixation cross for $1000 \mathrm{~ms}$. Subsequently, a prime arrow $\left(1.5^{\circ}\right.$ wide and $0.7^{\circ}$ high) pointing to the left or right was presented for $34 \mathrm{~ms}$ followed by a blank screen for $34 \mathrm{~ms}$. Then, a target arrow $\left(3.3^{\circ}\right.$ wide and $1.4^{\circ}$ high) pointing to the left or right was presented for $116 \mathrm{~ms}$ followed by a blank screen. Because the prime arrows fitted perfectly within the contours of the target arrow (i.e., metacontrast masking; Vorberg et al., 2003), primes were rendered invisible. This has the major advantage that task performance and metacognitive experiences are influenced without participants being aware of the visual stimuli driving these changes (Chambon and Haggard, 2012; Desender et al., 2014; Wenke et al., 2010). Participants were asked to respond as fast and accurate as possible to the direction of the target. They were instructed to press " $\mathrm{d}$ " in response to a left pointing target arrow and " $\mathrm{k}$ " in response to a right pointing target arrow with the middle finger of each hand on a qwerty keyboard. If a response to the target was registered within $3000 \mathrm{~ms}$, a blank screen was presented for $516 \mathrm{~ms}$, followed by a screen asking participants a metacognitive question: "How much difficulty did you experience when responding to the arrow?". They could answer either by pressing the "o" key with the ring finger of their right hand ("Rather more difficulty") or by pressing the " $m$ " key with the index finger of their right hand ("Rather less difficulty"). The wordings 'rather more' and 'rather less' were used in order to stress that the difference between both metacognitive experiences is small, a subtlety that is potentially lost when using the terms 'easy' versus 'difficult'. There was no time limit to answer this question. The inter-trial interval was $800 \mathrm{~ms}$.

Each participant started with 20 practice trials in which the metacognitive question was omitted. Subsequently, the experimenter explained that participants had to rate their experience associated with a trial after each response. The experimenter motivated participants to use all information available to them (i.e., difficulty, error-tendency, response fluency) to answer this question. Participants were informed that there would be an equal amount of "more difficult" and "less difficult" trials, and they were motivated to keep a balance between these responses. Participants received 20 additional practice trials with the metacognitive question. After these two training phases, each participant performed eight blocks of 80 trials each. In each block, half of the trials were congruent (i.e., prime and target pointing in the same direction), and half were incongruent (i.e., prime and target pointing in opposite directions) creating a response conflict.

Only after the main experiment, participants were informed about the presence of the primes, and participated in a subsequent detection task. In this task, participants were instructed to categorize the direction of the prime arrows, instead of the target arrows. During the detection task, targets were neutral with heads pointing in both directions to ensure that participants were not accidentally responding to the target. The detection task comprised of 100 trials. 


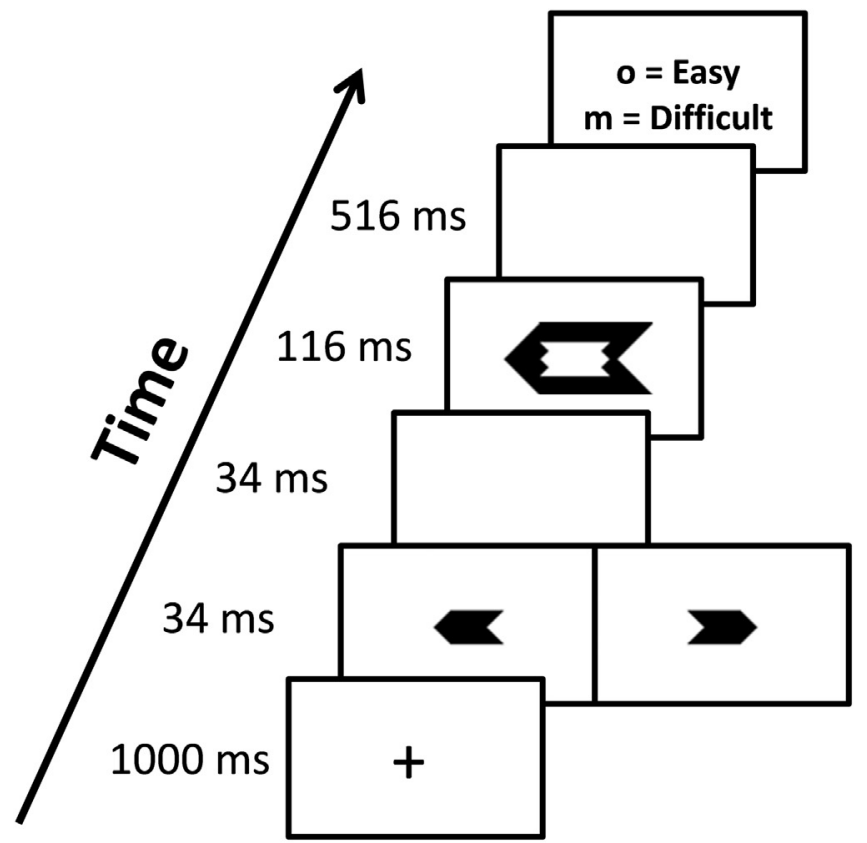

Main task

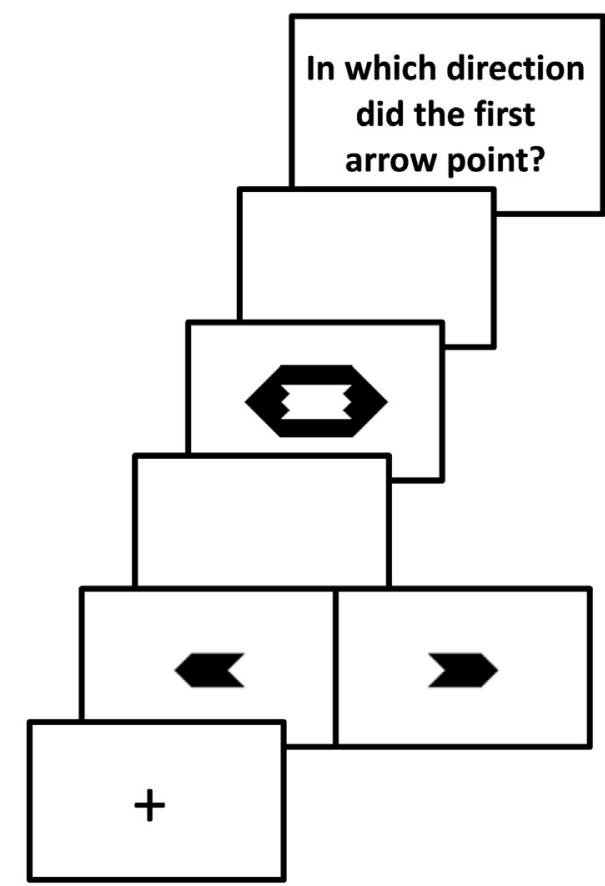

Detection task

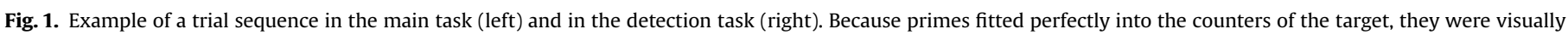
imperceptible.

\subsection{EEG recording and data pre-processing}

EEG data were recorded from 64 scalp locations (actiCHamp, Brain Products $\mathrm{GmbH}$, Germany) with a sample rate of $500 \mathrm{~Hz}$. Data were segmented from $-500 \mathrm{~ms}$ to $2000 \mathrm{~ms}$ relative to target onset. First, segments containing artefacts were identified by visual inspection and removed. Next, eyeblinks were removed using Independent Component Analysis (ICA), removing 1.47 components on average (range 1-2), and segments containing values $\pm 200 \mu \mathrm{V}$ were excluded using extreme value rejection. Bad (noisy) channels were replaced by an interpolated weighted average from surrounding electrodes using the EEGLAB toolbox (Delorme and Makeig, 2004) in Matlab. Finally, segments containing further artefacts, identified by visual inspection, were removed prior to averaging. For plotting purposes only, data were filtered using a $20 \mathrm{~Hz}$ low pass filter.

\subsection{Statistical approach}

In order to perform reliable analyses on our EEG data, a minimum number of 20 trials in each cell of the 2 by 2 interaction between congruency and metacognition was required. Due to the nature of our metacognitive measure, the data of nine participants contained less than 20 observations in at least one of these four cells $(M=6$, range $=0-15)$. For seven of them, this was caused by very high metacognitive performance (on average $79 \%$ correct classifications) leaving too few incorrect metacognitive responses. The other two participants showed a strong response bias, using almost exclusively the 'easy' option. Due to this unequal distribution, these participants were excluded from further analysis, leaving 15 participants in the final sample (five males, $M_{\text {age }}=24.5$ years, $S D=6.7$, range 19-42). Although the final sample size is sufficiently large to perform reliable analyses, one should keep in mind that this drop in sample size could potentially obscure some effects. To deal with this potential worry, we qualified our main
ERP findings by additionally computing the BayesFactor (BF) associated with each effect, using the default priors in the BayesFactor package (Morey and Rouder, 2014). Compared to classical $p$ values, a $B F$ has the advantage that it can dissociate between data in favor of the null hypothesis $(B F<1 / 3)$, data in favor of the alternative hypothesis $(B F>3)$ and data that is not informative ( $B F \approx 1$ ), (by Jeffreys's (1961), convention). As can be found below, our $B F$ results showed that for all non-significant $p$-values there was evidence in favor of the null, whereas for all significant $p$ values there was evidence for the alternative hypothesis. The only exception was the main effect of metacognition in the P3 time window ( $p=.08, B F=1.10$ ), where the $B F$ suggested that the data is not informative. To examine whether metacognitive experiences are related to the N2 and/or the P3, we first examined standard ERPs. To complement this approach, a more data-driven strategy was used in which a classifier was trained to predict the congruency status of individual trials, and subsequently it was tested if this classifier contained information about the metacognitive experience associated with each trial. Above and beyond the standard ERPs, this approach allows us to examine whether congruency information in the N2 and P3 is related to metacognitive experiences. Finally, source localization was used to confirm the relation between the ACC and the N2, and to investigate the neural origin of the P3.

\subsubsection{ERP analyses}

Our main analysis of averaged ERPs focused on the N2 and P3, which are successive deflections in the stimulus-locked ERP waveforms. Baseline activity was removed by subtracting the average voltage from -168 to -68 prior to target presentation (i.e., $100 \mathrm{~ms}$ period prior to prime onset). The time windows and spatial topography for the analysis of all ERP components were chosen based on a grand-average difference plot of congruency (incongruent-congruent), without taking metacognitive experience into account. As expected, this grand average showed a clear N2 
and P3 component. The N2 was computed across electrodes FCz and $\mathrm{Cz}$ in the $32 \mathrm{~ms}$ period around the peak of the component as measured in the grand average ERP (Clayson and Larson, 2011). This resulted in a time window between $246 \mathrm{~ms}$ and $278 \mathrm{~ms}$. The P3 was computed on electrodes $\mathrm{CPz}, \mathrm{Cz}$ and $\mathrm{FCz}$, in the $100 \mathrm{~ms}$ period around the moment where the congruency effect in the grand average ERP had its largest difference. This resulted in a time window between $360 \mathrm{~ms}$ and $460 \mathrm{~ms}$. Subsequently, mean amplitude was extracted from the specified time windows and the associated electrodes, separately for congruency (congruent versus incongruent) and metacognitive experience (easy versus difficult). These mean amplitudes were then submitted to two separate repeated measures ANOVAs (one for each time window), with congruency and metacognitive experience as within-subjects factors.

\subsubsection{Single-trial analysis}

To complement the standard ERP analysis, we also analyzed the data using a more data-driven approach. Based on the results from the ERP analysis (i.e., the interaction between congruency and metacognition in the P3; see below), we examined whether information about congruency on the single-trial level would predict the associated metacognitive experience. To achieve this, we used single-trial analyses and examined whether a classifier trained on congruency also contains information about metacognition. More specifically, the N2 and P3 components were analyzed on individual trials using the logistic-regression based linear derivation method introduced by Parra et al. (2005). An important advantage of this method is that it does not specify the spatial topography of the effects beforehand. This approach identifies the spatial distribution of scalp EEG activity in a given time window that maximally distinguishes two conditions (e.g., congruent versus incongruent) to deliver a scalar estimate of component amplitude on each trial. The derived estimates have a high signal-to-noise ratio because the discriminating component acts as a spatial filter, estimating component amplitude as a spatially weighted average across electrodes for each trial. The single-trial analysis was conducted separately for each participant by training a logistic regression classifier to discriminate congruency in either the N2 or the P3 time window. In a first step, the reliability of the classification was tested using a 10 -fold cross validation approach in which the classifier was trained on $90 \%$ of the trials and tested on the remaining $10 \%$. This procedure was repeated 10 times, on each occasion keeping a different $10 \%$ of the trials for testing. For each participant, we quantified our ability to successfully classify individual trials by calculating the $\mathrm{Az}$ score, which gives the area under the receiver operating characteristic (ROC) curve, derived from signal detection theory (e.g., Stanislaw and Todorov, 1999). To test for the significance of the single-trial classification, the distribution of this $\mathrm{Az}$ score was calculated by running 100 permutations of our classifier. This distribution was then compared to 100 permutations of $\mathrm{Az}$ scores using randomized condition labels, to provide an estimate of the null classification. A different randomization of the condition labels was carried out at each permutation. Group level analyses were performed by computing an average per subject over the permutations, and contrasting classifications made with true and random labels using a paired $t$-test. In a second step, the classifier was trained to predict congruency using all trials, and the resulting estimates were applied to all trials. Although this approach over-fits the data for congruency, it allows us to examine whether a classifier trained on predicting congruency on individual trials contains information about the metacognitive response.

\subsubsection{Source localization}

Based on the scalp-recorded electric potential distribution, the standardized low resolution brain electromagnetic tomography (sLORETA) software (http://www.uzh.ch/keyinst/loreta.htm) was used to compute the cortical three-dimensional distribution of current density (Fuchs et al., 2002; Pascual-Marqui et al., 2002). This method is based on the neurophysiological assumption of coherent co-activation of neighboring cortical areas, and computes the smoothest of all possible activity distributions (i.e., no a priori assumption is made on the number and locations of the sources). Note that, although there is a specific prediction that the N2 originates from the anterior cingulate cortex (Jiang et al., 2015; Van Veen and Carter, 2002), results from these analyses should be treated with caution given the limited spatial precision of EEG. This is especially the case for the $\mathrm{P} 3$, for which the a priori prediction is unclear. We conducted this method once on a difference wave of the N2 time window measuring congruency (i.e., Incongruent-Congruent), and once on a difference wave in the P3 time window measuring the interaction in the P3 (Easy (Incongruent-Congruent)-Difficult ${ }_{\text {(Incongruent-Congruent). To analyze these difference }}$ waves, we used a paired-groups analysis, and tested the N2 and P3 time windows in the corresponding difference waves, using $t$ statistics with the variance smoothing parameter set to zero. Finally, 5000 permutations were performed to compute correct thresholds for $t$ values in order to test for significance at an alpha level of .05 . These computations are performed in a realistic head model using the MNI152 template, with the three-dimensional solution space restricted to cortical gray matter. The intracerebral volume is partitioned in 6239 voxels at $5 \mathrm{~mm}$ spatial resolution.

\section{Results}

\subsection{Behavioral results}

\subsubsection{Metacognitive experience of difficulty}

Because performance in responding to the target arrow was at ceiling level (96.7\%) and metacognitive responses to incorrect trials are quite trivial, we deleted error trials (3.3\%), trials following an error (3.1\%) and the first trial of each block (1.2\%) from all following analyses. Then, it was examined whether participants' metacognitive experiences (i.e., whether a trial was as experienced as easy or difficult), coincided with the actual congruency, which would show evidence of above chance-level metacognitive performance. Note that one should be careful not to confuse basic task performance (which was at ceiling level) and this measure of metacognitive performance. We used the data of the main experiment to calculate a $d^{\prime}$ based on signal detection theory (henceforth called conflict- $d$ ), which provides a measure of metacognitive performance while controlling for response bias (Green and Swets, 1966). Incongruent trials were treated as signal, and congruent trials were treated as noise. The "difficult" response was considered a hit on incongruent trials and a false alarm on congruent trials. Hit proportions were computed by dividing the total number of hits by the number of signals, and false alarm proportions were computed by dividing the total number of false alarms by the number of trials where no signal was present. Mean conflict- $d$ was 0.54 , showing significant above chance level metacognitive performance, $t(14)=3.01, p=.009$. Participants correctly classified congruent trials as easy and incongruent trials as difficult in $59 \%$ of the trials. Trials judged to be easy were more frequently congruent (36.9\%) than incongruent (26.9\%), whereas trials judged to be difficult were more frequently incongruent (22.4\%) than congruent (13.8\%). Next, to test if metacognitive accuracy is based on participants' capacity to introspect on their own RTs (Marti et al., 2010), a subset of the data was selected in which RTs for congruent and incongruent trials were matched. For each congruent trial, an incongruent trial was randomly selected whose 
A.
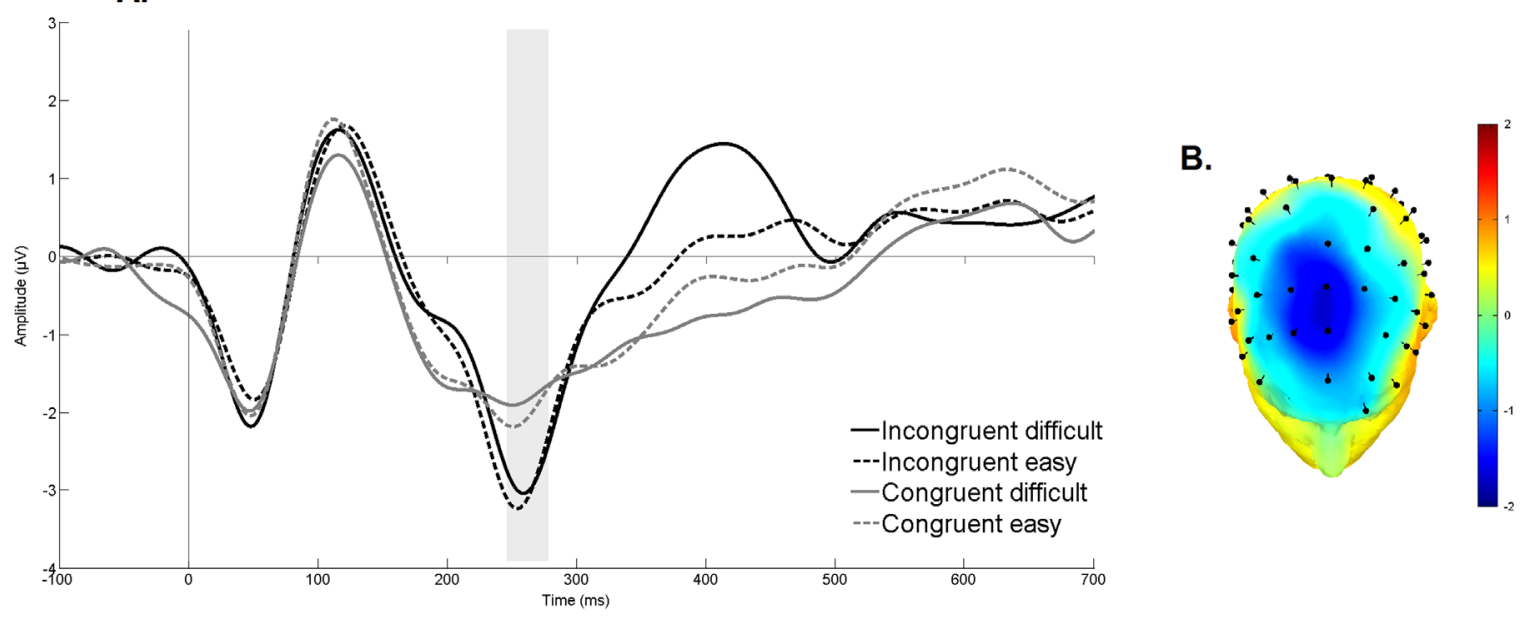

C.
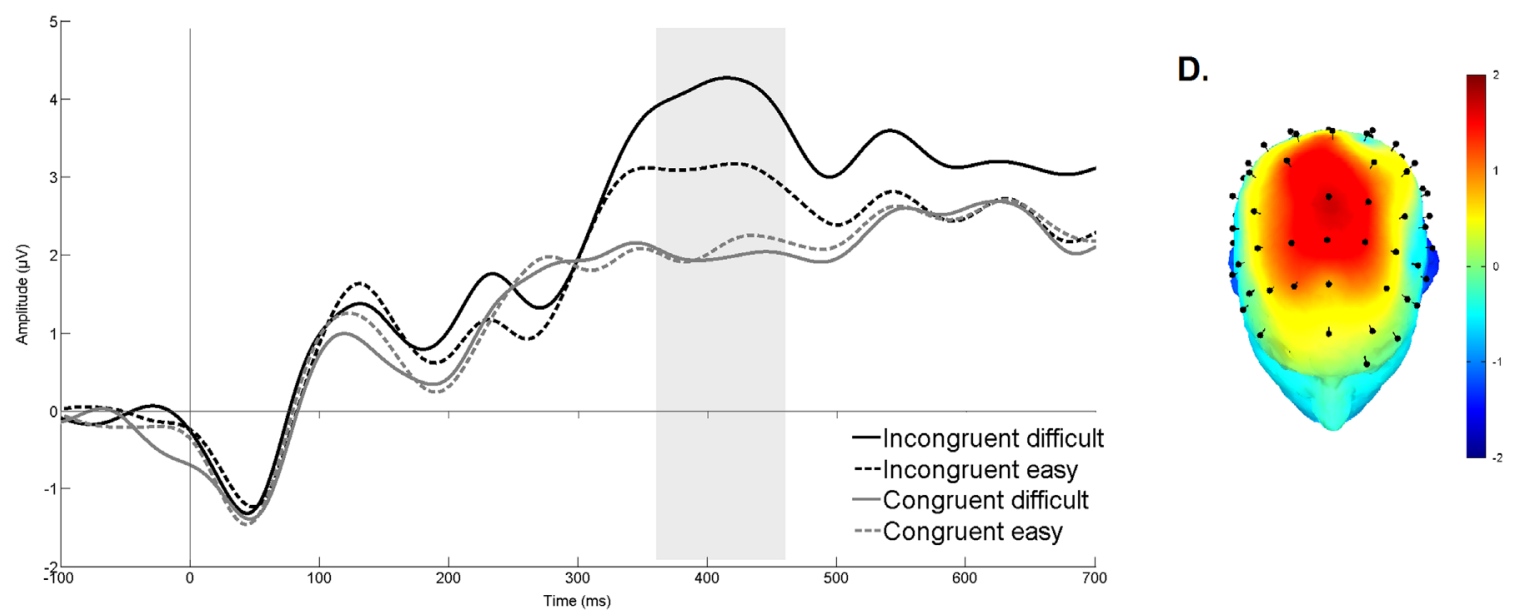

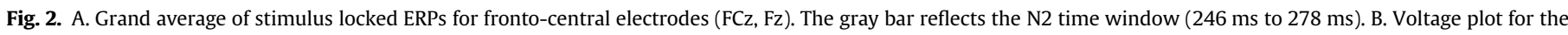

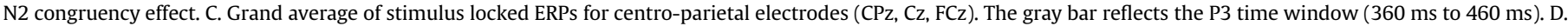
Voltage plot for the P3 congruency effect.

RT deviated $5 \mathrm{~ms}$ maximally. Trials that could not be matched across conditions were discarded. In this RT-matched set, metacognitive performance was still reliably above chance level, conflict- $d=.45, t(14)=2.56, p=.023$, with participants classifying $57 \%$ of the trials correctly. This finding shows that participants were able to introspect on the presence of response conflict, even in the absence of differences in response time. ${ }^{1}$

To confirm that this above chance-level categorization is not caused by prime perception, prime visibility was assessed using the data of the detection task. A $d^{\prime}$ measure (treating left pointing primes as signal) was calculated and this measure did not deviate from chance level (i.e., zero), $d^{\prime}=-0.019, t(14)=-0.25, p=.81$, indicating that participants were completely unable to dissociate left from right pointing primes. Furthermore, no correlation was found between conflict- $d$ and prime visibility, $\beta_{1}=-0.361, t$ $(14)=-0.57, p=.57$, showing that participants' metacognitive capacity was independent from prime visibility. A positive significant intercept, $\quad \beta_{0}=.53, \quad t(14)=2.89, \quad p=.013$, was observed,

\footnotetext{
${ }^{1}$ When analyzing the complete sample $(N=31)$, these behavioral results were similar. Metacognitive performance was above chance level ( $64 \%$ correct), conflict$d=.92, t(30)=4.79, p<.001$. This was also the case when congruent and incongruent trials were matched for response time (61\% correct, conflict- $d=.79, t(30)=$ $4.40, p<.001)$. Finally, RTs still showed both the main effect of congruency, $F$ $(1,30)=56.84, p<.001$ and of metacognitive response, $F(1,30)=27.01, p<.001$.
}

demonstrating that even when prime visibility was statistically zero, we still observed above chance level metacognitive performance (Greenwald et al., 1995).

\subsubsection{Target responses}

To analyze the responses to the target arrow, median RTs were submitted to a 2 (congruency: congruent or incongruent) $\times 2$ (metacognitive response: easy or difficult) repeated measures ANOVA. A main effect of congruency was observed, $F(1,14)=18.46$, $p<.001$ : RTs were faster on congruent (445 ms) than incongruent trials (486 ms). There was also a significant main effect of metacognitive response, $F(1,14)=12.28, p=.004$, indicating that trials labeled as difficult were responded to slower $(521 \mathrm{~ms})$ than trials labeled as easy ( $465 \mathrm{~ms}$ ). The interaction between both factors was not significant, $F<1{ }^{2}$

\subsection{Electrophysiological results}

\subsubsection{Stimulus locked ERPS}

Fig. 2A and $\mathrm{C}$ present the grand average stimulus-locked ERPs

\footnotetext{
${ }^{2}$ This same analysis on the error rates yielded no significant effect of congruency, $F(1,14)=3.11, p=.10$, a trivial main effect of metacognition, $F(1,14)=12.56$ $p=.003$, and no interaction, $p>.27$.
} 
A.

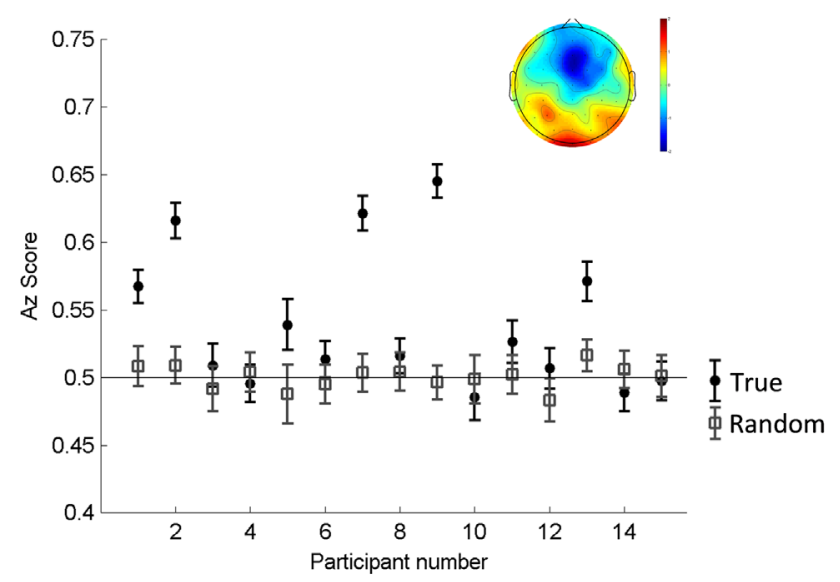

B.

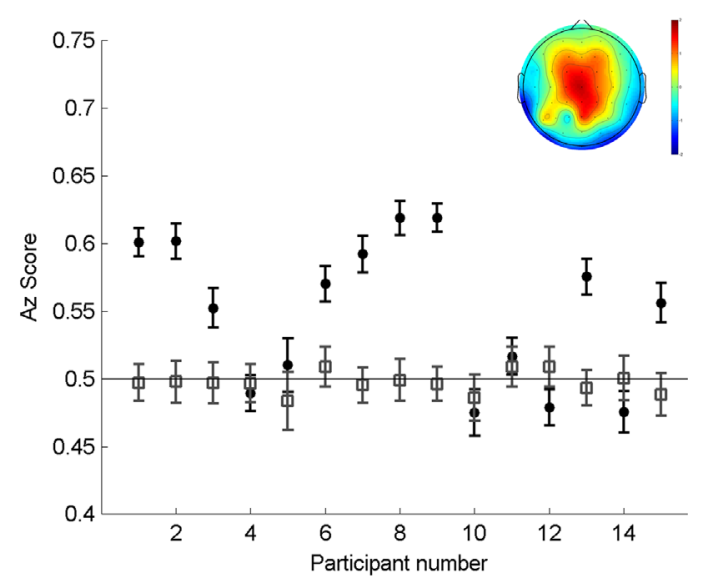

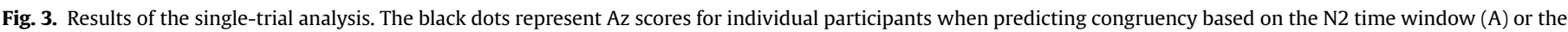

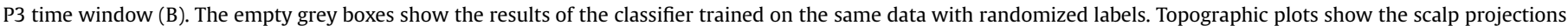
obtained from the logistic regression classifier, averaged over subjects. Error bars reflect $95 \%$ confidence intervals.

for fronto-central and centro-parietal electrodes, respectively, dependent on congruency and metacognitive experience. We observed an N2 in a time window between $246 \mathrm{~ms}$ and $278 \mathrm{~ms}$ poststimulus, located at fronto-central electrodes (FCz, Fz; see Fig. 2B), followed by a P3 in a time window between $360 \mathrm{~ms}$ and $460 \mathrm{~ms}$ post-stimulus, located around central electrodes (CPz, Cz, FCz; see Fig. 2D).

A repeated measures ANOVA with the factors congruency (congruent or incongruent) and metacognitive experience (easy or difficult) on the mean average voltage during the $\mathrm{N} 2$ time window across electrodes $\mathrm{FCZ}$ and $\mathrm{Fz}$ indeed showed a main effect of congruency, $F(1,14)=10.47, p=.005, B F=8.52$. The amplitude of incongruent trials was more negative than that of congruent trials. Crucially, there was no main effect of metacognition, $F<1$, $B F=0.29$, nor an interaction between both factors, $F<1, B F=0.28$. A post-hoc $t$-test showed that even the small difference between incongruent trials judged to be easy and incongruent trials judged to be difficult that can be seen in Fig. 2A was far from significant, $p>.80, B F=0.27$. Exactly the same results were found when the procedure described above to control for differences in RTs was applied.

The same repeated measure ANOVA on the P3 time window on the average of the $\mathrm{CPz}, \mathrm{Cz}$ and $\mathrm{FCz}$ similarly showed a main effect of congruency, $F(1,14)=35.25, p<.001, B F=702.17$. The amplitude of incongruent trials was more positive than that of congruent trials. The main effect of metacognition was close to significance, $F$ $(1,14)=3.63, p=.08, B F=1.10$. Crucially, there was a significant interaction between both factors, $F(1,14)=8.01, p=.013, B F=4.40$, showing that the effect of metacognition on the P3 was limited to incongruent trials. Post-hoc $t$-tests showed that incongruent trials judged to be difficult and incongruent trials judged to be easy differed both from each other and from all other trial types (all $p^{\prime} s<.018$, all $B F ' s>3.42$ ). Only the difference between easy and difficult congruent trials was not significant $(p=.65, B F=0.29)$. Again, the same pattern of results was observed in an RT-matched subset of the data, indicating that our results reflect metacognitive experiences associated with response fluency, and not just a mere read-out of reaction times. ${ }^{3}$

\footnotetext{
${ }^{3}$ Note that our results can also not be explained by a difference in signal-tonoise ratio between the four conditions. When randomly selecting trials from each condition until the number of observations was matched, (separately for each participant), exactly the same pattern of results was obtained.
}

\subsubsection{Single-trial analysis}

First, to get an indication of the robustness of our classifier, we examined the classification $(\mathrm{Az})$ scores for each participant when predicting congruency based on the N2 (Fig. 3A) and the P3 (Fig. 3B) time-window. On the individual level, we were able to classify significantly better compared to random classification for 8 of 15 participants when predicting the N2, and for 9 of 15 participants when predicting the $\mathrm{P} 3$. On a group level, both predictions for the N2, $t(14)=3.08, p=.008$, and the P3, $t(14)=3.25$, $p=.005$, were significantly better than random classification. As can be seen in Fig. 3, this data-driven approach gives a topography that is highly consistent with the ERP analysis. Hence, this singletrial analysis provides us with reliable classification performance.

Next, it was examined whether a classifier trained on all data to predict congruency on individual trials contains information about the metacognitive response. As such, we extracted congruency probabilities associated with each trial from the classifier, and submitted these to a repeated measures ANOVA, with the factors congruency and metacognitive response as within-subject factors. For the P3 time window, this analysis showed the trivial main effect of congruency, $F(1,14)=329.06, p<.001$, and importantly, an interaction with metacognitive response, $F(1,14)=5.64, p=.032$. For incongruent trials, the probability scores significantly differentiated between easy and difficult trials, $t(14)=3.25, p=.005$, whereas this was not the case for congruent trials, $p>.60$. For the $\mathrm{N} 2$ time window, this analysis showed only the trivial main effect of congruency, $F(1,14)=236.87, p<.001$, but no interaction with metacognitive report, $p>.19$. Hence, congruency information on the individual trial level in the $\mathrm{N} 2$ time window does not contain information with regard to the metacognitive response (see Fig. 4).

\subsubsection{Source localization}

Our ERP results make a strong case for the P3, but not the N2, being significantly modulated by metacognitive experience. Next, we examined whether we could link particular brain areas to these components. First, we examined the neural generator of the congruency effect in the N2. Given numerous previous reports that this component reflects a sensitivity of the anterior cingulate cortex (ACC) to competing response demands (Carter and van Veen, 2007), we first wanted to replicate this finding. In Fig. 5A, it can be seen that this prediction is confirmed by the data. A cluster of voxels in the ACC is more responsive to incongruent compared to congruent trials. Note, however, that only the maximum peak of activation in this contrast was significant at the .05 level (MNI coordinates $(x, y, z): 10,25,35)$. 

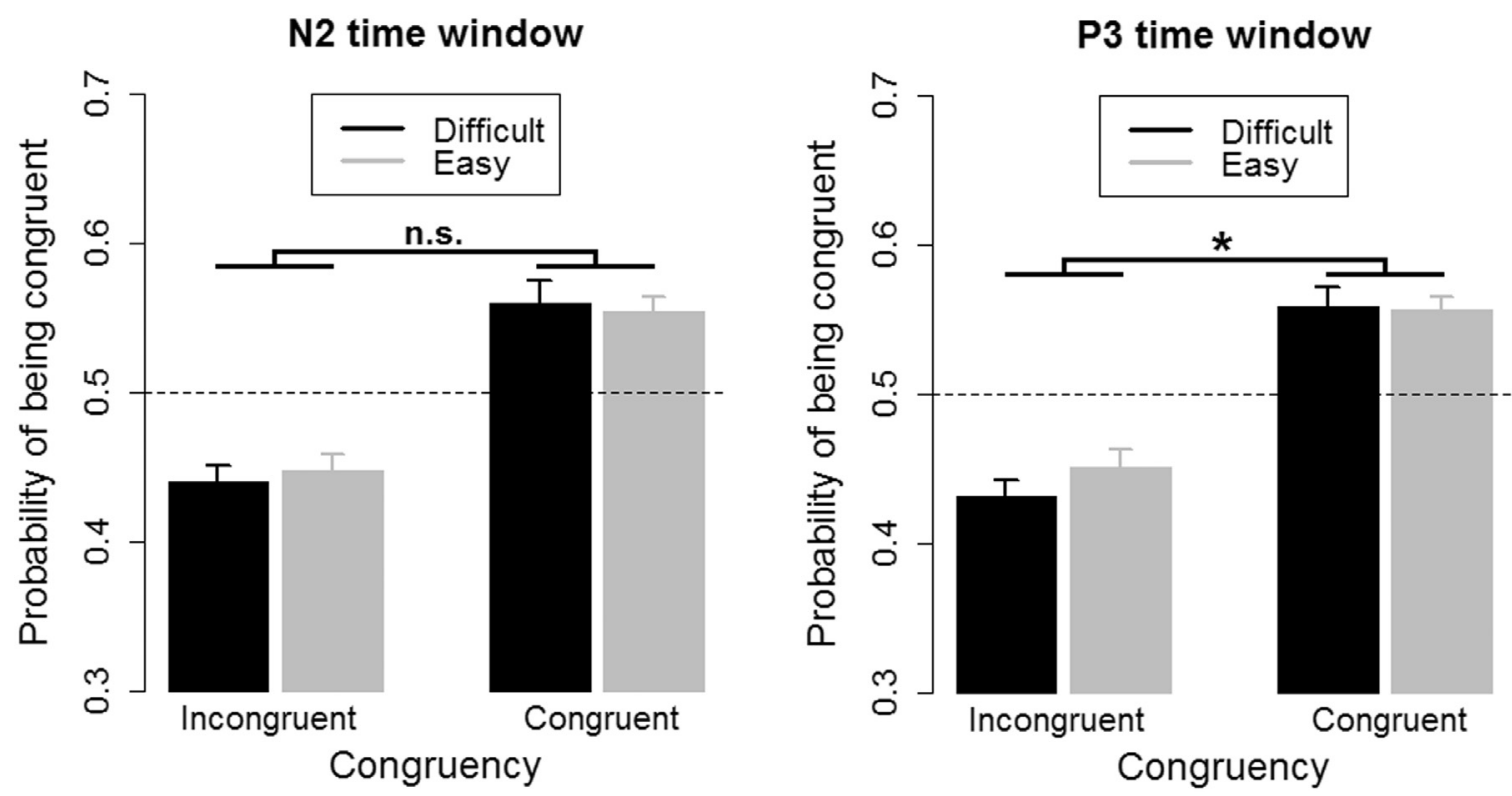

Fig. 4. Congruency probabilities, separated by metacognitive response. Error bars reflect $95 \%$ confidence intervals.

A.

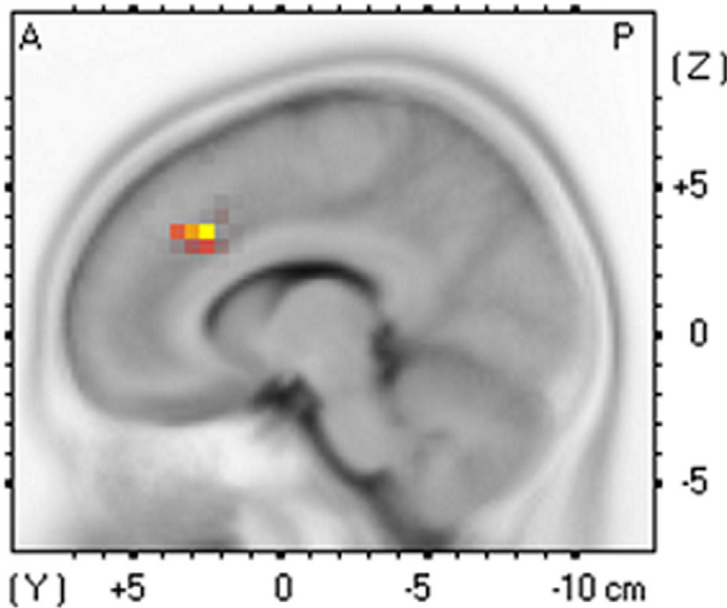

B.

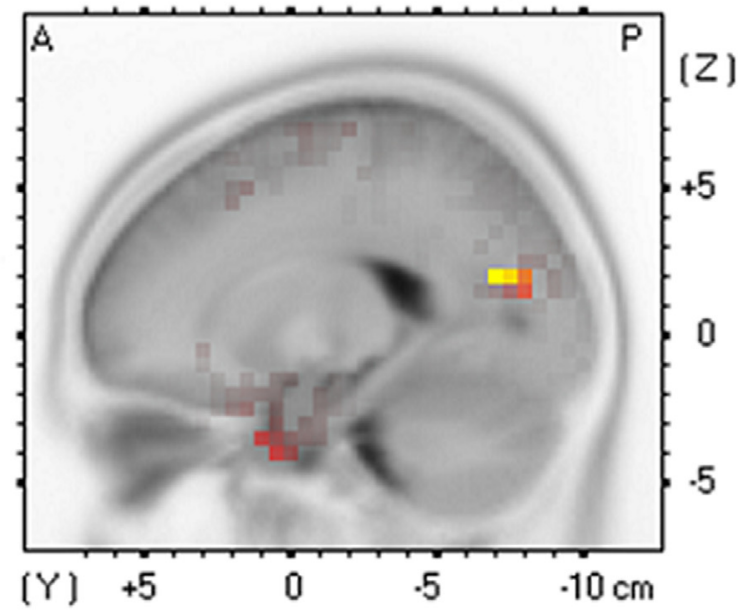

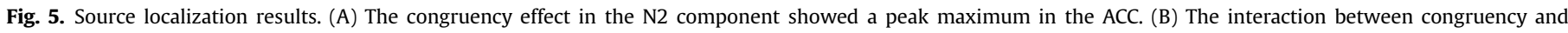
metacognition in the $\mathrm{P} 3$ component showed a peak maximum in the left precuneus.

Second, we examined the neural correlates of the metacognitive experiences on the P3 component. Because our ERP analysis showed that the effect of metacognition was restricted to incongruent trials, we computed a single difference waveform representing this interaction (Difficult (Incongruent-Congruent) $^{-}$ Easy(Incongruent-Congruent)). In Fig. 5B, it can be seen that the peak activation of this contrast is in the left precuneus (MNI coordinates $(x, y, z):-20,-70,20)$. Apart from this peak, several other voxels also reached significance at the .05 level (see Table 1 ). Note that the extent of these significant areas was quite small, calling for extra caution when interpreting these results. Therefore, we only tentatively conclude that the interaction between congruency and metacognitive experience is correlated with activity in the (left) precuneus.

\subsection{Link between behavioral and electrophysiological results}

Based on our results, we can conclude that the P3 component is crucially linked to metacognitive experiences. If this is the case, a relation between behavioral performance in the metacognitive task (as indexed by conflict- $d$ ) and the magnitude of the interaction in the P3 component (reflecting that the effect of metacognition was restricted to incongruent trials) should be expected. A correlational analysis confirmed this, $R^{2}=.32, \beta=1.247, t(14)=$ $2.47, p=.028$, see Fig. 6 . The better participants were able to behaviorally dissociate easy from difficult trials, the larger the interaction between congruency and metacognition on the P3 component $\quad\left(\mathrm{P}_{\text {(congruency }}{ }_{\text {metacognition })}=\right.$ Easy $_{(\text {Incongruent-Congruent }}{ }^{-}$

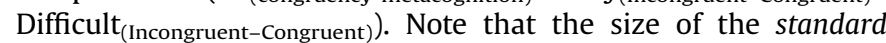
conflict-P3 component ( $\mathrm{P} 3_{\text {(Incongruent-Congruent) }}$ ) was not predictive of metacognitive performance, $p=.56$.

\section{Discussion}

The current study is the first report on the temporal dynamics underlying metacognition. In a masked priming paradigm, we 
Table 1

Voxels showing a significant activation at the .05 level $(t>4.078)$ for the interaction between congruency and metacognition in the P3.

\begin{tabular}{|c|c|c|c|c|}
\hline \multicolumn{3}{|c|}{ MNI coordinates } & \multirow[t]{2}{*}{$t$ Value } & \multirow[t]{2}{*}{ Anatomical region } \\
\hline$X$ & $Y$ & $Z$ & & \\
\hline-20 & -70 & 20 & 4.93 & Precuneus \\
\hline-20 & -75 & 20 & 4.86 & Precuneus \\
\hline-20 & -80 & 20 & 4.58 & Cuneus \\
\hline 40 & -20 & 45 & 4.40 & Precentral Gyrus \\
\hline-5 & 30 & 60 & 4.37 & Superior Frontal Gyrus \\
\hline-20 & -80 & 15 & 4.36 & Cuneus \\
\hline 40 & -20 & 50 & 4.29 & Precentral Gyrus \\
\hline 5 & 55 & 40 & 4.23 & Medial Frontal Gyrus \\
\hline-20 & 5 & -40 & 4.23 & Uncus \\
\hline 40 & -20 & 65 & 4.21 & Precentral Gyrus \\
\hline 55 & -25 & 35 & 4.20 & Postcentral Gyrus \\
\hline-20 & 10 & -35 & 4.19 & Superior Temporal Gyrus \\
\hline 45 & -20 & 65 & 4.18 & Postcentral Gyrus \\
\hline
\end{tabular}

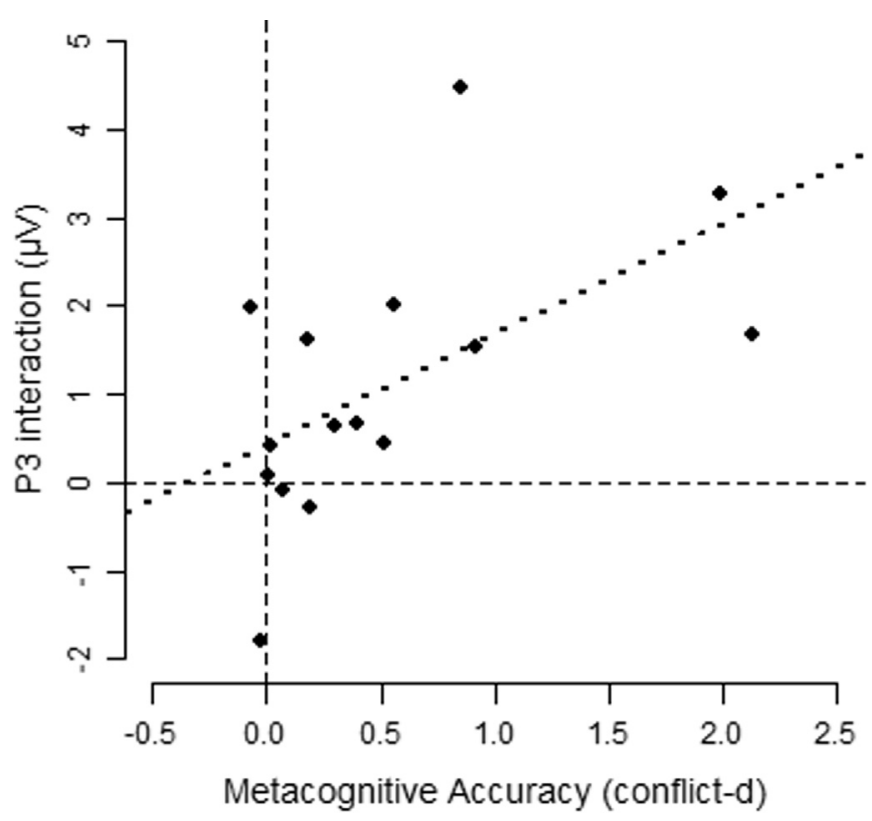

Fig. 6. Relation between metacognitive performance (conflict- $d$ ) and the size of the modulation between congruency and metacognitive response in the P3 component.

induced metacognitive experiences of difficulty by subliminally priming the correct or incorrect response. Because primes were imperceptible, metacognitive experiences do not reflect metacognition about visual perception, but rather introspection on subtle differences in task performance. As expected, participants were able to reliably dissociate easy (i.e., congruent) from difficult (i.e., incongruent) trials. Our ERPs showed that congruency modulated both the N2 and the P3 component. Crucially, only in the P3, congruency was modulated by the metacognitive experience of difficulty. Complementing this finding, using single-trial analysis, we showed that the magnitude of the P3 modulation by congruency on individual trials was predictive of the metacognitive response. Source localization pointed to the precuneus as the neural generator of metacognitive experiences, although this finding should be treated with caution given the limited spatial precision of EEG. In the remainder, we will discuss the interpretation and significance of our results.

\subsection{The P3 and metacognition}

Conflict tasks are known to reliably modulate both the N2 and the P3 component (Ullsperger et al., 2014). Interestingly, we did not find any support for a role of the N2 in metacognitive experiences. This suggests that the involvement of the ACC is confined to task-related processes, whereas it is independent of later metacognitive experiences. This finding can aid the interpretation of ACC activation in studies examining the neural correlates of metacognition (e.g., Fleming et al., 2012). This result also adds to the lively debate on the role of awareness in the detection of response conflicts and response errors, as reflected by the N2 and ERN components, respectively. Some have argued that these components cannot be observed when the presence of response conflict (e.g., Dehaene et al., 2003) or response errors (e.g., Scheffers and Coles, 2000; Shalgi and Deouell, 2012) remains below the threshold of awareness, whereas others have challenged this claim (e.g., Nieuwenhuis et al., 2001; O'Connell et al., 2007). As discussed by Wessel (2012), part of this discrepancy might stem from methodological differences between studies. In particular, studies on error awareness frequently make use of an extra 'awareness-button' that needs to be pressed in the case of an error, which might not be sensitive enough to detect subtle differences in error awareness. In the current work, participants rather rated their metacognitive experience on each trial, which is a more appropriate approach to measure metacognition. Nevertheless, we found evidence that the N2 is sensitive to unconscious response conflict, independent of participant's metacognitive awareness (e.g., Jiang et al., 2015).

As mentioned in the introduction, the P3 has already been ascribed several different functions, such as stimulus evaluation (Coles et al., 1985; Purmann et al., 2011) or the engagement of attentional resources (Clayson and Larson, 2011; West, 2003). However, because previous studies did not assess metacognitive experiences, they were not able to determine whether the P3 varies as a function of stimulus characteristics, such as response conflict, or as a function of subjective experience (but see Del Cul et al. (2007)). Based on the current data, we can conclude that in conflict tasks the P3 component is actually an expression of the interplay between task-related parameters (such as response conflict) and metacognitive experiences. Our analysis indicated that even on individual trials, the size of the modulation of the P3 component by congruency was predictive of whether participants experienced it as an easy or a difficult trial. This was especially the case for incongruent trials, which is reasonable given that only on incongruent trials there is consistent motor conflict of which participants can become aware. Congruent trials, on the other hand, do not (by definition) contain motor conflict, and hence congruent trials that are experienced as difficult have to result from other sources than response conflict. It could for example be that being distracted during a congruent trial makes you label this trial as "difficult". While being distracted can lead to difficult responses on congruent trials, it can never lead to an easy response on incongruent trials, hence it selectively affects congruent trials. One obvious way to test this distraction hypothesis is to examine whether RTs to the main target arrow were different on congruent trials that were judged as easy compared to congruent trials that were judged difficult. We observed that responses were indeed reliably faster on congruent trials judged as easy (444 ms versus $503 \mathrm{~ms}$ ), $t(14)=2.79, p=.014$, suggesting that response time serves as a proxy for task difficulty (Kiani et al., 2014). This finding is interesting because, as mentioned in the Section 3.2.1, the effect of congruency on metacognitive experience was also present in a subset of the data that was matched for RTs, suggesting that congruency and RT are two independent sources affecting metacognitive experiences, with only the former modulating the P3. 
Moreover, note that the EEG waveform associated with congruent trials labeled as easy or difficult were not different from each other at any other moment in the epoch (see Fig. 2C), strengthening our hypothesis that some source of distraction can lead to "difficult" judgments on congruent trials. As a final piece of evidence in support of this suggestion, RTs to the metacognitive question were indeed much slower to difficult congruent trials $(M=528 \mathrm{~ms}$ postresponse) than to easy congruent trials ( $M=422 \mathrm{~ms}$ post-response), $p=.03$, whereas this was not the case on incongruent trials, $p=.60$. In sum, we conclude that our P3 modulation might only reflect metacognitive awareness caused by response conflict, but not by other sources such as distractions.

\subsection{The neural correlate of metacognition}

Interestingly, source localization pointed to the precuneus as the neural generator of the modulation by metacognition in the P3. Although source localization results of EEG with 64 electrodes should be treated very cautiously, it is nevertheless interesting to note that this finding fits well within current literature on metacognition. Although initial functional MRI studies pointed to the anterior PFC as neural correlate of visual metacognition (Fleming et al., 2010; Yokoyama et al., 2010), recent studies have found the precuneus to be related to metacognition in the memory domain (Baird, Smallwood, Gorgolewski and Margulies, 2013; McCurdy et al., 2013). Rather than stressing this difference in modality (i.e., visual versus memory), these findings can easily be integrated with the current results by assuming that the functional role of the precuneus is to focus attention on the target of metacognition. A crucial aspect in our task was that metacognitive judgments were about the experience of task difficulty. Thus, in order to accurately detect which experience they had on each trial, participants needed to orient their attention internally, which has been linked to activity in the precuneus (Vanhaudenhuyse et al., 2011). The metacognitive experiences in our task might also be highly related to self-consciousness and self-reflection (Cavanna and Trimble, 2006; Vogt and Laureys, 2005). In line with this suggestion, a recent study argued that the precuneus may contain common mechanisms for different types of metacognition (visual and memory; McCurdy et al., 2013), a proposal which fits well with our suggestion that the P3 component reflects the internal orientation of attention. In order to provide more convincing support for our claim linking metacognitive experiences and the precuneus, future studies should aim to replicate this finding using more precise brain imaging techniques, such as fMRI.

\subsection{The functional role of the P3}

Our data provide a compelling case for a role of the P3 in metacognition, for which we see two potential explanations in terms of its functional role. First, the most straightforward explanation is that the P3 reflects a neural correlate of metacognitive awareness. Support for this comes from several studies claiming that the P3 is a neural correlate of conscious access (Del Cul et al., 2007; Kouider et al., 2013), studies claiming that the Pe (which has been linked to the P3; Ridderinkhof et al., 2009) reflects error awareness (Nieuwenhuis et al., 2001), and a recent study linking the experience of agency to the P3 component (Kühn et al., 2011). This latter observation seems of particular interest, given that our methodological approach bears close resemblance to a line of research on the sense of agency (Chambon and Haggard, 2012; Wenke et al., 2010). Here, the same subliminal priming paradigm is used, but responses are additionally followed by a stimulus, whose color is entirely predicted by prime-target congruency. Participants are found to experience a larger sense of agency over colors that follow congruent trials compared to colors following incongruent trials. Hence, response congruency influences the subjective experience of agency. Given the resemblance between this research line and ours, the involvement of the P3 component in both our study and recent work on the sense of agency (Kühn et al., 2011), might point to a general role of the P3 in metacognitive experiences.

Alternatively, it can also be that the modulation of the P3 component by metacognition is actually a precursor of metacognition, providing input for metacognitive experiences. Evidence for this possibility comes from a recent study on error awareness that observed a relation between the magnitude of the Pe and the criterion that participants had set to signal their errors (Steinhauser and Yeung, 2010). They found that a high criterion to signal an error resulted in a larger magnitude of the Pe than a low criterion. From this, the authors concluded that the Pe component reflects an evidence accumulator sensitive to the amount of evidence that an error was committed, rather than the neural underpinning of error awareness itself. This is in line with the interpretation of our source localization results, suggesting that the modulation in the $\mathrm{P} 3$ reflects the focusing of attention on internal information that enables metacognition. Note, however, that this comparison should be treated with caution. Error rates in conflict tasks are typically below $15 \%$ and are known to produce large orienting behaviors (Notebaert et al. 2009; Jessup et al. 2010), so simply generalizing the functional role of the Pe to that of the P3 might be too simplistic.

Finally, an open question for future research will be to examine which specific processes, apart from congruency between prime and target, determine whether a particular trial will be experienced as either easy or difficult. We examined the possibility that differences in response selection might underlie these metacognitive experiences. Another interesting area for further examination is the role of expectancy in metacognitive experiences of difficulty (e.g., Gratton et al., 1988). For example, expecting a particular response (e.g., left) can lead to an 'easy' experience when this expectation is met, and to a 'difficult' experience when it is not met. Likewise, expecting a target stimulus at a given moment in time can lead to an easy experience when this temporal expectation is met, and to a difficult experience when the stimulus is unexpectedly presented earlier or later in time. Interestingly, it could be argued that the mechanism by which expectations influence metacognitive experiences is identical to that examined in the current study. Expecting a particular response to occur, can lead to pre-activation of this response. When this expectation is not met, there will be a response conflict between the expected response and the correct response. This competition between incompatible responses will influence both the performance (i.e., better performance on expected responses) and the metacognitive experience (i.e., 'easy' experience on a predicted response). This very same mechanism can explain the results of the current study. In our study, when an invisible prime triggers the incorrect response, this creates the (unconscious) expectation of the incorrect response (Chambon and Haggard, 2012), leading to a response conflict between the primed and the correct response. This competition between two incompatible responses will lead to a reduction in performance and a 'difficult' metacognitive experience. Thus, despite the different origin of the response conflict, the same mechanism of competition between conflicting responses might underlie the modulation of performance and metacognition, both when the conflict is induced by the expectation of a particular response, or when this is induced by means of a priming procedure. Future studies could aim to consistently manipulate expectancy, in order to examine whether the P3 can also be linked to metacognition when using a different manipulation to influence metacognitive experiences. 


\section{Conclusion}

In the current study, we used EEG to dissociate task-related activity from later metacognitive processes. We observed that both the N2 and P3 component were modulated by congruency, but only in the P3 we observed an interaction with metacognition. We conclude that the N2 component reflects only task-related activation, whereas the P3 component reflects an interplay between task-related activation and metacognitive experiences.

\section{Acknowledgments}

This work was supported by Grants of the Research Foundation Flanders, Belgium (FWO-Vlaanderen) awarded to K.D. (Grant number $11 \mathrm{H} 3415 \mathrm{~N}$ ) and to E.V.D.B. (Grant number G023213N). F.V. O. is a scientific collaborator of the F.R.S.-FNRS (Belgium). F.V.O. was partially supported by European Research Council (ERC) Grant 340718 to Axel Cleeremans. The authors declare no conflict of interest.

\section{References}

Baird, B., Smallwood, J., Gorgolewski, K.J., Margulies, D.S., 2013. Medial and lateral networks in anterior prefrontal cortex support metacognitive ability for memory and perception. J. Neurosci. 33 (42), 16657-16665. http://dx.doi.org/ 10.1523/JNEUROSCI.0786-13.2013.

Boldt, A., Yeung, N., 2015. Shared neural markers of decision confidence and error detection. J. Neurosci. 35 (8), 3478-3484. http://dx.doi.org/10.1523/ JNEUROSCI.0797-14.2015.

Carter, C.S., van Veen, V., 2007. Anterior cingulate cortex and conflict detection: an update of theory and data. Cogn. Affect. Behav. Neurosci. 7 (4), 367-379.

Cavanna, A.E., Trimble, M.R., 2006. The precuneus: a review of its functional anatomy and behavioural correlates. Brain: J. Neurol. 129 (Pt 3), 564-583. http: //dx.doi.org/10.1093/brain/awl004.

Chambon, V., Haggard, P., 2012. Sense of control depends on fluency of action selection , not motor performance. Cognition 125 (3), 441-451. http://dx.doi.org/ 10.1016/j.cognition.2012.07.011.

Charles, L., Van Opstal, F., Marti, S., Dehaene, S., 2013. Distinct brain mechanisms for conscious versus subliminal error detection. NeuroImage 73, 80-94. http://dx. doi.org/10.1016/j.neuroimage.2013.01.054.

Clayson, P.E., Larson, M.J., 2011. Conflict adaptation and sequential trial effects: support for the conflict monitoring theory. Neuropsychologia 49 (7), 1953-1961. http://dx.doi.org/10.1016/j.neuropsychologia.2011.03.023.

Coles, M.G.H., Gratton, G., Bashore, T.R., Eriksen, C.W., Donchin, E., 1985. A psychophysiological investigation of the continuous flow model of human information processing. J. Exp. Psychol.: Hum. Percept. Perform. 11 (5), 529-561.

de Gardelle, V., Mamassian, P., 2014. Does confidence use a common currency across two visual tasks? Psychol. Sci. 25 (6), 1286-1288. http://dx.doi.org 10.1177/0956797614528956.

Dehaene, S., Artiges, E., Naccache, L., Martelli, C., Viard, A., Schürhoff, F., Martinot, J.L., 2003. Conscious and subliminal conflicts in normal subjects and patients with schizophrenia: the role of the anterior cingulate. Proc. Natl. Acad. Sci. USA 100 (23), 13722-13727. http://dx.doi.org/10.1073/pnas.2235214100.

Del Cul, A., Baillet, S., Dehaene, S., 2007. Brain dynamics underlying the nonlinear threshold for access to consciousness. PLoS Biol. 5 (10), e260. http://dx.doi.org 10.1371/journal.pbio.0050260.

Delorme, A., Makeig, S., 2004. EEGLAB: an open source toolbox for analysis of single-trial EEG dynamics including independent component analysis. J. Neurosci. Methods 134 (1), 9-21. http://dx.doi.org/10.1016/j.jneumeth.2003.10.009.

Desender, K., Van Opstal, F., Van den Bussche, E., 2014. Feeling the conflict: the crucial role of conflict experience in adaptation. Psychol. Sci. 6 (25), 375-383. http://dx.doi.org/10.1177/0956797613511468.

Díaz, F., Lindín, M., Galdo-Alvarez, S., Facal, D., Juncos-Rabadán, O., 2007. An eventrelated potentials study of face identification and naming: the tip-of-the-tongue state. Psychophysiology 44 (1), 50-68. http://dx.doi.org/10.1111/ j.1469-8986.2006.00483.x.

Fleming, S.M., Huijgen, J., Dolan, R.J., 2012. Prefrontal contributions to metacognition in perceptual decision making. J. Neurosci. 32 (18), 6117-6125. http://dx. doi.org/10.1523/JNEUROSCI.6489-11.2012.

Fleming, S.M., Lau, H.C., 2014. How to measure metacognition. Front. Hum. Neurosci. 8, 1-9. http://dx.doi.org/10.3389/fnhum.2014.00443.

Fleming, S.M., Ryu, J., Golfinos, J.G., Blackmon, K.E., 2014. Domain-specific impairment in metacognitive accuracy following anterior prefrontal lesions. Brain 137 (10), 2811-2822. http://dx.doi.org/10.1093/brain/awu221.

Fleming, S.M., Weil, R.S., Nagy, Z., Dolan, R.J., Rees, G., 2010. Relating introspective accuracy to individual differences in brain structure. Science 329 (5998),
1541-1543. http://dx.doi.org/10.1126/science.1191883.

Fuchs, M., Kastner, J., Wagner, M., Hawes, S., Ebersole, J.S., 2002. A standardized boundary element method volume conductor model. Clin. Neurophysiol.: Off. J. Int. Fed. Clin. Neurophysiol. 113 (5), 702-712.

Gratton, G., Coles, M.G., Sirevaag, E.J., Eriksen, C.W., Donchin, E., 1988. Pre- and poststimulus activation of response channels: a psychophysiological analysis. J. Exp. Psychol.: Hum. Percept. Perform. 14 (3), 331-344. http://dx.doi.org/ 10.1037/0096-1523.14.3.331.

Green, D.M., Swets, J.A., 1966. Signal Detection Theory and Psychophysics. Wiley, New York.

Greenwald, a G., Klinger, M.R., Schuh, E.S., 1995. Activation by marginally perceptible ("subliminal") stimuli: dissociation of unconscious from conscious cognition. J. Exp. Psychol.: Gen. 124 (1), 22-42.

Hillman, K.L., Bilkey, D.K., 2013. Persisting through subjective effort: a key role for the anterior cingulate cortex? Behav. Brain Sci. 36 (6), 707-726.

Hughes, G., Yeung, N., 2011. Dissociable correlates of response conflict and error awareness in error-related brain activity. Neuropsychologia 49 (3), 405-415. http://dx.doi.org/10.1016/j.neuropsychologia.2010.11.036.

Jeffreys, H., 1961. The Theory of Probability, 3rd ed. Oxford University Press, Oxford.

Jessup, R.K., Busemeyer, J.R., Brown, J.W., 2010. Error effects in anterior cingulate cortex reverse when error likelihood is high. J. Neurosci. 30 (9), 3467-3472. http://dx.doi.org/10.1523/JNEUROSCI.4130-09.2010.

Jiang, J., Zhang, Q., van Gaal, S., 2015. Conflict awareness dissociates theta-band neural dynamics of the medial frontal and lateral frontal cortex during trial-bytrial cognitive control. NeuroImage 116, 102-111. http://dx.doi.org/10.1016/j. neuroimage.2015.04.062.

Kiani, R., Corthell, L., Shadlen, M.N., 2014. Choice certainty is informed by both evidence and decision time. Neuron 84 (6), 1329-1342. http://dx.doi.org/ 10.1016/j.neuron.2014.12.015.

Kouider, S., Stahlhut, C. Gelskov, S.V., Barbosa, L.S., Dutat, M., de Gardelle, V., Dehaene-Lambertz, G., 2013. A neural marker of perceptual consciousness in infants. Science 340 (6130), 376-380. http://dx.doi.org/10.1126/science.1232509.

Kühn, S., Nenchev, I., Haggard, P., Brass, M., Gallinat, J., Voss, M., 2011. Whodunnit? electrophysiological correlates of agency judgements. PLoS ONE 6 (12), 1-6. http://dx.doi.org/10.1371/journal.pone.0028657.

Marti, S., Sackur, J., Sigman, M., Dehaene, S., 2010. Mapping introspection's blind spot: reconstruction of dual-task phenomenology using quantified introspection. Cognition 115 (2), 303-313. http://dx.doi.org/10.1016/j. cognition.2010.01.003.

McCurdy, L.Y., Maniscalco, B., Metcalfe, J., Liu, K.Y., de Lange, F.P., Lau, H., 2013. Anatomical coupling between distinct metacognitive systems for memory and visual perception. J. Neurosci. 33 (5), 1897-1906. http://dx.doi.org/10.1523/ JNEUROSCI.1890-12.2013.

Metcalfe, J., Finn, B., 2008. Evidence that judgments of learning are causally related to study choice. Psychon. Bull. Rev. 15 (1), 174-179. http://dx.doi.org/10.3758/ PBR.15.1.174.

Morey, R.D., Rouder, J.N., 2014. BayesFactor: Computation of Bayes factors for common design. Retrieved from 〈http://cran.r-project.org/ package $=$ BayesFactor $\rangle$.

Nieuwenhuis, S., Ridderinkhof, K.R., Blom, J., Band, G.P.H., Kok, A., 2001. Error-related brain potentials are differentially related to awareness of response errors: evidence from an antisaccade task. Psychophysiology 38 (5), 752-760.

Notebaert, W., Houtman, F., Opstal, F., Van, Gevers, W., Fias, W., Verguts, T., 2009. Post-error slowing: an orienting account. Cognition 111 (2), 275-279. http://dx. doi.org/10.1016/j.cognition.2009.02.002.

O'Connell, R.G., Dockree, P.M., Bellgrove, M. a, Kelly, S.P., Hester, R., Garavan, H., Foxe, J.J., 2007. The role of cingulate cortex in the detection of errors with and without awareness: a high-density electrical mapping study. Eur. J. Neurosci. 25 (8), 2571-2579. http://dx.doi.org/10.1111/j.1460-9568.2007.05477.x.

Parra, L.C., Spence, C.D., Gerson, A.D., Sajda, P., 2005. Recipes for the linear analysis of EEG. Neurolmage 28 (2), 326-341. http://dx.doi.org/10.1016/j. neuroimage.2005.05.032.

Pascual-Marqui, R.D., Esslen, M., Kochi, K., Lehmann, D., 2002. Functional imaging with low-resolution brain electromagnetic tomography (LORETA): a review. Methods Find. Exp. Clin. Pharmacol. 24 (Suppl. C), S91-S95.

Purmann, S., Badde, S., Luna-Rodriguez, A., Wendt, M., 2011. Adaptation to frequent conflict in the eriksen flanker task. J. Psychophysiol. 25 (2), 50-59. http://dx.doi. org/10.1027/0269-8803/a000041.

Ridderinkhof, K.R., Ramautar, J.R., Wijnen, J.G., 2009. To P(E) or not to P(E): a P3-like ERP component reflecting the processing of response errors. Psychophysiology 46 (3), 531-538. http://dx.doi.org/10.1111/j.1469-8986.2009.00790.x.

Scheffers, M.K., Coles, M.G., 2000. Performance monitoring in a confusing world: error-related brain activity, judgments of response accuracy, and types of errors. J. Exp. Psychol.: Hum. Percept. Perform. 26 (1), 141-151. http://dx.doi.org/ 10.1037/0096-1523.26.1.141.

Shackman, A.J., Salomons, T.V., Slagter, H. a, Fox, A.S., Winter, J.J., Davidson, R.J., 2011. The integration of negative affect, pain and cognitive control in the cingulate cortex. Nat. Rev. Neurosci. 12 (3), 154-167. http://dx.doi.org/10.1038/ nrn2994.

Shalgi, S., Deouell, L.Y., 2012. Is any awareness necessary for an Ne? Front. Hum. Neurosci. 6, 1-15. http://dx.doi.org/10.3389/fnhum.2012.00124.

Spunt, R.P., Lieberman, M.D., Cohen, J.R., Eisenberger, N.I., 2012. The phenomenology of error processing: the dorsal ACC response to stop-signal errors tracks reports of negative affect. J. Cognit. Neurosci. 24 (8), 1753-1765. http://dx.doi. org/10.1162/jocn_a_00242.

Stanislaw, H., Todorov, N., 1999. Calculation of signal detection theory measures. 
Behav. Res. Methods Instrum. Comput. 31 (1), 137-149.

Steinhauser, M., Yeung, N., 2010. Decision processes in human performance monitoring. J. Neurosci: Off. J. Soc. Neurosci. 30 (46), 15643-15653. http://dx.doi. org/10.1523/JNEUROSCI.1899-10.2010.

Ullsperger, M., Fischer, A.G., Nigbur, R., Endrass, T., 2014. Neural mechanisms and temporal dynamics of performance monitoring. Trends Cognit. Sci. 18 (5), 259-267. http://dx.doi.org/10.1016/j.tics.2014.02.009.

Van Veen, V., Carter, C.S., 2002. The timing of action-monitoring processes in the anterior cingulate cortex. J. Cognit. Neurosci. 14 (4), 593-602. http://dx.doi.org/ 10.1162/08989290260045837.

Vanhaudenhuyse, A., Demertzi, A., Schabus, M., Noirhomme, Q., Bredart, S., Boly, M., Laureys, S., 2011. Two distinct neuronal networks mediate the awareness of environment and of self. J. Cognit. Neurosci. 23 (3), 570-578.

Vogt, B. a, Laureys, S., 2005. Posterior cingulate, precuneal and retrosplenial cortices: cytology and components of the neural network correlates of consciousness. Prog. Brain Res. 150, 205-217. http://dx.doi.org/10.1016/S0079-6123 (05)50015-3.

Vorberg, D., Mattler, U., Heinecke, A., Schmidt, T., Schwarzbach, J., 2003. Different time courses for visual perception and action priming. Proc. Natl. Acad. Sci. USA 100 (10), 6275-6280. http://dx.doi.org/10.1073/pnas.0931489100.
Wenke, D., Fleming, S.M., Haggard, P., 2010. Subliminal priming of actions influences sense of control over effects of action. Cognition 115 (1), 26-38. http://dx doi.org/10.1016/j.cognition.2009.10.016.

Wessel, J.R., 2012. Error awareness and the error-related negativity: evaluating the first decade of evidence. Front. Hum. Neurosci. 6, 1-16. http://dx.doi.org/ 10.3389/fnhum.2012.00088.

West, R., 2003. Neural correlates of cognitive control and conflict detection in the Stroop and digit-location tasks. Neuropsychologia 41 (8), 1122-1135.

Yeung, N., Botvinick, M.M., Cohen, J.D., 2004. The neural basis of error detection: conflict monitoring and the error-related negativity. Psychol. Rev. 111 (4), 931-959. http://dx.doi.org/10.1037/0033-295X.111.4.931.

Yeung, N., Summerfield, C., 2012. Metacognition in human decision-making: confidence and error monitoring. Philos. Trans. R. Soc. Lond. 367 (1594), 1310-1321. http://dx.doi.org/10.1098/rstb.2011.0416.

Yokoyama, O., Miura, N., Watanabe, J., Takemoto, A., Uchida, S., Sugiura, M., Nakamura, K., 2010. Right frontopolar cortex activity correlates with reliability of retrospective rating of confidence in short-term recognition memory performance. Neurosci. Res. 68 (3), 199-206. http://dx.doi.org/10.1016/j. neures.2010.07.2041. 